Björn Ivarsson · Jerk Matero

\title{
The blow-up rate of solutions to boundary blow-up problems for the complex Monge-Ampère operator
}

Received: 5 December 2005

Published online: 9 June 2006

Abstract. A regularity result for solutions to boundary blow-up problems for the complex Monge-Ampère operator in balls in $\mathbb{C}^{n}$ is proved. For certain boundary blow-up problems on bounded, strongly pseudoconvex domains in $\mathbb{C}^{n}$ with smooth boundary an estimate of the blow-up rate of solutions are given in terms of the distance to the boundary and the product of the eigenvalues of the Levi form.

\section{Introduction}

Let $\Omega$ be a bounded strongly pseudoconvex domain in $\mathbb{C}^{n}$ with smooth boundary. By smooth we mean $C^{\infty}$-smooth. We want to study the problem

$$
\left\{\begin{array}{l}
\operatorname{det}\left(\frac{\partial^{2} u}{\partial z_{j} \partial \bar{z}_{k}}(z)\right)=f(z, u(z)) \text { in } \Omega, \\
\lim _{z \rightarrow z_{0}} u(z)=\infty \text { for all } z_{0} \in \partial \Omega,
\end{array}\right.
$$

where $f$ satisfies some regularity and growth conditions. The special case

$$
f(z, u(z))=k(z) \exp (K u(z))
$$

for a constant $K>0$ and $k(z)$ a strictly positive smooth function on $\Omega$ has been studied by Cheng and Yau [3]. They showed that for this type of right-hand side there is a unique smooth plurisubharmonic solution. Their motivation for solving this problem was to construct Kähler-Einstein metrics. We shall briefly outline how a solution of such a Monge-Ampère equation implies the existence of a Kähler-Einstein metric. Remember that a Hermitian metric $d s^{2}=\sum_{j, k=1}^{n} h_{j \bar{k}}(z) \mathrm{d} z_{j} \otimes \mathrm{d} \bar{z}_{k}$ has an associated form $\omega=(i / 2) \sum_{j, k=1}^{n} h_{j \bar{k}}(z) \mathrm{d} z_{j} \wedge \mathrm{d} \bar{z}_{k}$. The metric $\mathrm{d} s^{2}$ is said to be a Kähler metric if $\mathrm{d} \omega=0$ and $\omega$ is said to be a Kähler form. A plurisubharmonic function $u$ gives rise to a Hermitian metric with $\omega=\partial \bar{\partial} u$. In fact, this is a Kähler metric since $\mathrm{d} \omega=(\partial+\bar{\partial}) \partial \bar{\partial} u=\bar{\partial} \partial \bar{\partial} u=-\partial \bar{\partial} \bar{\partial} u=0$. A metric is said to be an Einstein metric if it's Ricci curvature tensor is a constant multiple of the metric

B. Ivarsson ( $\varangle)$ : Mathematisches Institut, Universität Bern, Sidlerstrasse 5, 3012 Bern, Switzerland. e-mail: bjoern.ivarsson@math.unibe.ch

J. Matero: E. Öhman J:or Fondkommision, P.O. Box 7415, 10391 Stockholm, Sweden. e-mail: jerk.matero@ohman.se

Mathematics Subject Classification (2000): 32W20 $\cdot 32$ A25 $\cdot 35$ B40 $\cdot 35 J 60$ 
tensor. Curvature tensors are really defined in terms of connections and are in some sense independent of the metric. However, given a metric there is a choice of connection so that the connection is said to be compatible with the metric. In the case of a complex manifold there is also the concept of a connection being compatible with the complex structure. It is know that on a complex manifold with Hermitian metric there is a unique connection which is compatible with both the metric and the complex structure. With this choice of connection the Ricci curvature tensor is given by

$$
-\sum_{j, k=1}^{n} \frac{\partial^{2}}{\partial z_{j} \partial \bar{z}_{k}} \log \left(\operatorname{det}\left(\frac{\partial^{2} u}{\partial z_{j} \partial \bar{z}_{k}}\right)\right) \mathrm{d} z_{j} \otimes \mathrm{d} \bar{z}_{k} .
$$

A good reference for this is Kobayashi's book [8]. We see that a plurisubharmonic solution of Problem (1) with right-hand side

$$
f(z, u(z))=\mathrm{e}^{K u(z)},
$$

satisfies

$$
\log \left(\operatorname{det}\left(\frac{\partial^{2} u}{\partial z_{j} \partial \bar{z}_{k}}\right)\right)=K u(z),
$$

and hence gives rise to a metric which is both Kähler and Einstein, a Kähler-Einstein metric. Also since $u(z)$ tends to infinity at the boundary the metric is complete.

In this paper we give a description of how fast the solutions of Problem (1) tend to $\infty$ as $z$ approaches boundary points. We shall sometimes refer to this as the blow-up rate of the solution. In Sect. 4 we apply our results to describe the boundary behavior of the Bergman kernel.

Caffarelli, Kohn, Nirenberg and Spruck proved the following theorem in [2] and it will be of great importance for our construction.

Theorem 1.1. Let $\Omega$ be a bounded, strongly pseudoconvex domain in $\mathbb{C}^{n}$ with smooth boundary. Let $f \in C^{\infty}(\bar{\Omega} \times \mathbb{R})$ be a strictly positive function which is increasing in the second variable. Let $\varphi \in C^{\infty}(\partial \Omega)$. Then the problem

$$
\left\{\begin{array}{l}
\operatorname{det}\left(\frac{\partial^{2} u}{\partial z_{j} \partial \bar{z}_{k}}\right)=f(z, u(z)) \text { in } \Omega, \\
u=\varphi \text { on } \partial \Omega
\end{array}\right.
$$

has a unique strictly plurisubharmonic solution $u$. Moreover we have $u \in C^{\infty}(\bar{\Omega})$.

We will also need the following lemma, again from [2].

Lemma 1.2. Let $\Omega$ be a bounded domain in $\mathbb{C}^{n}$ and suppose that $v, w \in C^{\infty}(\bar{\Omega}) \cap$ $\mathcal{P S H}(\Omega)$. Assume that

$$
\left\{\begin{array}{l}
\operatorname{det}\left(\frac{\partial^{2} v}{\partial z_{j} \partial \bar{z}_{k}}\right) \geq \operatorname{det}\left(\frac{\partial^{2} w}{\partial z_{j} \partial \bar{z}_{k}}\right) \quad \text { in } \Omega \\
\text { and } v \leq w \text { on } \partial \Omega
\end{array}\right.
$$

Then $v \leq w$ in $\Omega$. 
The following comparison principle is sometimes useful. For a proof see for example [6].

Lemma 1.3. Let $\Omega$ be a bounded pseudoconvex domain in $\mathbb{C}^{n}$. Assume that $f: \Omega \times$ $\mathbb{R} \rightarrow \mathbb{R}$ is a nonnegative function which is increasing in the second variable. Let $v, w \in C^{\infty}(\bar{\Omega}) \cap \mathcal{P} \mathcal{S H}(\Omega)$ and $u \in C^{\infty}(\Omega) \cap \mathcal{P S H}(\Omega)$ such that $\lim _{z \rightarrow z_{0}} u(z)=$ $\infty$ for all $z_{0} \in \partial \Omega$. Then

(i) $\operatorname{det}\left(\partial^{2} w / \partial z_{j} \partial \bar{z}_{k}\right) \leq f(z, w(z)), f(z, v(z)) \leq \operatorname{det}\left(\partial^{2} v / \partial z_{j} \partial \bar{z}_{k}\right)$ and $v \leq w$ on $\partial \Omega$ implies that $v \leq w$ in $\Omega$ and

(ii) $\operatorname{det}\left(\partial^{2} u / \partial z_{j} \partial \bar{z}_{k}\right) \leq \bar{f}(z, u(z)), f(z, v(z)) \leq \operatorname{det}\left(\partial^{2} v / \partial z_{j} \partial \bar{z}_{k}\right)$ implies that $v \leq u$ in $\Omega$.

If we combine Theorem 1.1 and Lemma 1.3 we get a comparison principle for solutions to Problem (1) and Problem (2).

Corollary 1.4. Let $\Omega$ be a bounded, strongly pseudoconvex domain in $\mathbb{C}^{n}$ with smooth boundary and assume that $\varphi$ and $\psi \in C^{\infty}(\partial \Omega)$. Assume that $f \in C^{\infty}(\bar{\Omega} \times$ $\mathbb{R})$ is a strictly positive function which is increasing in the second variable. Let $v$ and $w$ be plurisubharmonic solutions to Problem (2) smooth on $\bar{\Omega}$ with boundary values $\varphi$ and $\psi$ respectively. Then

(i) if $\varphi \leq \psi$ on $\partial \Omega$ we have $v \leq w$ in $\Omega$ and

(ii) if $u$ is a smooth plurisubharmonic solution to Problem (1) we have $w \leq u$ in $\Omega$.

Apart for the conditions put on $f$ in Theorem 1.1 we shall often assume:

(A) There exists functions $h \in C^{\infty}(\bar{\Omega})$ and $f_{1} \in C^{\infty}(\mathbb{R})$ and two strictly positive constants $c_{1}$ and $c_{2}$ such that

$$
\lim _{t \rightarrow+\infty} \frac{f(z, t)}{f_{1}(t)}=h(z)
$$

uniformly in $\Omega$ and $c_{1} f_{1}(t) \leq f(z, t) \leq c_{2} f_{1}(t)$ for all $(z, t) \in \Omega \times \mathbb{R}$.

(B) The function $f_{1}$ is strictly positive and increasing.

(C) The function

$$
\Psi_{n}(a)=\int_{a}^{\infty}((n+1) F(y))^{-1 /(n+1)} \mathrm{d} y
$$

exists for $a>0$, where $F^{\prime}(s)=f_{1}(s)$ and $F(0)=0$.

Regularity and uniqueness questions for Problem (1) are quite delicate. We shall prove Proposition 2.1, a regularity result in balls. Uniqueness will not be dealt with at all. These questions are studied by the first author in [7]. 


\section{Existence of solutions}

We shall study the problem

$$
\left\{\begin{array}{l}
\operatorname{det}\left(\frac{\partial^{2} v}{\partial z_{j} \partial \bar{z}_{k}}\right)=k(|z|) f_{1}(v(z)) \text { in } B_{R}(0) \\
\lim _{|z| \rightarrow R} v(z)=\infty
\end{array}\right.
$$

where $k:[0, R] \rightarrow\left[c_{1}, c_{2}\right]$ is a smooth function which satisfies $k^{(2 l+1)}(0)=0$ for all $l \in \mathbb{N}$ and $0<c_{1} \leq c_{2}<\infty$. We require that derivatives of odd order vanishes at 0 because we want the function $k(|z|)$ to be smooth at the origin.

Proposition 2.1. Let $R, c_{1}$ and $c_{2}$ be strictly positive real numbers such that $c_{1} \leq$ $c_{2}$. Assume that $k:[0, R] \rightarrow\left[c_{1}, c_{2}\right]$ is a smooth function such that $k^{(2 l+1)}(0)=0$ for all $l \in \mathbb{N}$. Suppose that $f_{1} \in C^{\infty}(\mathbb{R})$ satisfies assumptions $(\mathrm{B})$ and $(\mathrm{C})$. Then Problem (3) has a smooth solution. Moreover the solution is radial.

Before we prove Proposition 2.1 we state some results that we shall use in the proof. The following result was proved in [6].

Proposition 2.2. Assume that $\Omega$ is a bounded convex domain in $\mathbb{C}^{n}$ and that $K$ a compact subset of $\Omega$. Let $\varphi: \partial \Omega \rightarrow \mathbb{R}$ be a nonpositive function and $g \in C^{\infty}(\bar{\Omega} \times$ $\mathbb{R})$ be a strictly positive function which is increasing in the second variable. Assume that $w \in C^{\infty}(\bar{\Omega}) \cap \mathcal{P S H}(\Omega)$ is a solution of

$$
\left\{\begin{array}{l}
\operatorname{det}\left(\frac{\partial^{2} w}{\partial z_{j} \partial \bar{z}_{k}}\right)=g(z, w(z)) \text { in } \Omega \\
w(z)=\varphi(z) \text { on } \partial \Omega
\end{array}\right.
$$

Let $D$ be the diameter of $\Omega$ and

$$
C=\sup \left(\left|\frac{\partial g^{1 / n}}{\partial x_{l}}(z, t)\right| ;(z, t) \in \Omega \times\left[\inf _{z \in \Omega} w(z), 0\right] \text { and } l=1, \ldots, 2 n\right) \text {. }
$$

Then there exists a compact set $L$ satisfying $K \Subset L \Subset \Omega$ so that, given

$$
M=\sup \left(\left|\min \left\{0, \frac{\partial w}{\partial v}(\zeta)\right\}\right| ; \zeta \in \partial \Omega, z \in L \text { and } v=(\zeta-z) /|\zeta-z|\right) \text {, }
$$

we have

$$
\begin{array}{r}
\sup \left(\left|\frac{\partial w}{\partial x_{l}}(z)\right| ; z \in K\right) \leq C D^{2} \\
+\frac{2 \sup _{z \in K}|w(z)|+2 \sup _{z \in \partial \Omega}|\varphi(z)|+2 D M+C D^{3}}{\inf _{z \in K} d_{\Omega}(z)}
\end{array}
$$

for $l=1, \ldots, 2 n$. 
We shall also use Proposition 2.3 below which was proved by Błocki in [1]. Here we write

$$
\begin{aligned}
\|u\|_{C^{1}(\Omega)}= & \sup (|u(z)| ; z \in \Omega) \\
& +\sum_{j=1}^{n}\left(\sup \left(\left|\frac{\partial u}{\partial z_{j}}(z)\right| ; z \in \Omega\right)+\sup \left(\left|\frac{\partial u}{\partial \bar{z}_{j}}(z)\right| ; z \in \Omega\right)\right)
\end{aligned}
$$

and for $0<\alpha<1$

$$
\|u\|_{C^{\alpha}(\Omega)}=\sup \left(\frac{|u(z)-u(w)|}{|z-w|^{\alpha}} ; z, w \in \Omega, z \neq w\right) .
$$

Proposition 2.3. Let $w$ be a $C^{4}$ plurisubharmonic function in an open set $\Omega$ in $\mathbb{C}^{n}$ and $\psi(z)=\operatorname{det}\left(\partial^{2} w / \partial z_{j} \partial \bar{z}_{k}(z)\right)$. Assume that for some nonnegative $K_{0}, K_{1}, b, B_{0}$ and $B_{1}$ we have

$$
\|w\|_{C^{1}(\Omega)} \leq K_{0}, \quad \sup _{\Omega} \Delta w(z) \leq K_{1}
$$

and

$$
b \leq \psi(z) \leq B_{0},\left\|\psi^{1 / n}(z)\right\|_{C^{1}(\Omega)} \leq B_{1} .
$$

Then for any $\Omega^{\prime} \Subset \Omega$ there are two constants $\alpha$ and $C$ where $\alpha \in(0,1)$ depends only on $n, K_{0}, K_{1}, b, B_{0}$ and $B_{1}$, and $C$ depends, besides those quantities, on $\inf _{\Omega^{\prime}} d_{\Omega}(z)$, such that

$$
\sup \left(\left\|\frac{\partial^{2} w}{\partial z_{j} \partial \bar{z}_{k}}(z)\right\|_{C^{\alpha}\left(\Omega^{\prime}\right)} ; j, k=1, \ldots, n\right) \leq C .
$$

We are now ready to prove the result.

Proof. (Proposition 2.1) Let $u_{N}$ be solutions of Problem (2) with right-hand side $f(z, u)=k(|z|) f_{1}(u), \Omega=B_{R}(0)$ and $\varphi \equiv N$. Let $\Phi_{A}(z)=A z$ for Hermitian matrices $A$ which satisfies $\operatorname{det} A=1$. We have $\Phi_{A}\left(B_{R}(0)\right)=B_{R}(0)$ and if $w=\Phi_{A}(z)$

$$
\begin{aligned}
\operatorname{det}\left(\frac{\partial^{2}\left(u_{N} \circ \Phi_{A}\right)}{\partial z_{j} \partial \bar{z}_{k}}(z)\right) & =(\operatorname{det} A)^{2} \operatorname{det}\left(\frac{\partial^{2} u_{N}}{\partial w_{j} \partial \bar{w}_{k}}(w)\right) \\
& =\operatorname{det}\left(\frac{\partial^{2} u_{N}}{\partial w_{j} \partial \bar{w}_{k}}(w)\right) .
\end{aligned}
$$

Lemma 1.3 gives that $u_{N}$ is a radial function and also that $u_{N} \leq u_{N+1}$. Put $u(z)=$ $\lim _{N \rightarrow \infty} u_{N}(z)$. First we shall construct a function $v$ which satisfies $u_{N} \leq v$ for all $N$. This will guarantee that $u$ exists.

Let $\rho(z)=K\left(|z|^{2}-R^{2}\right)$, where $K$ is a constant which will be chosen later, and assume that $h: \mathbb{R}^{-} \rightarrow \mathbb{R}$ be a strictly increasing convex function which satisfies $\lim _{x \rightarrow 0-} h(x)=\infty$. Put $v=h \circ \rho$. Then

$$
\frac{\partial v}{\partial z_{j}}=K \bar{z}_{j} h^{\prime}(\rho), \quad \frac{\partial v}{\partial \bar{z}_{k}}=K z_{k} h^{\prime}(\rho)
$$


and

$$
\frac{\partial^{2} v}{\partial z_{j} \partial \bar{z}_{k}}=K \delta_{j k} h^{\prime}(\rho)+K z_{k} \bar{z}_{j} h^{\prime \prime}(\rho) .
$$

A calculation yields

$$
\begin{aligned}
\operatorname{det}\left(\frac{\partial^{2} v}{\partial z_{j} \partial \bar{z}_{k}}\right) & =K^{n}\left(h^{\prime}(\rho)^{n}-|z|^{2} h^{\prime \prime}(\rho) h^{\prime}(\rho)^{n-1}\right) \\
& =K^{n}\left(\frac{1}{\left(h^{-1}\right)^{\prime}(v)^{n}}-|z|^{2} \frac{\left(h^{-1}\right)^{\prime \prime}(v)}{\left(h^{-1}\right)^{\prime}(v)^{n+2}}\right) .
\end{aligned}
$$

If we choose $h^{-1}(v)=-\Psi_{n}(v)$ we get

$$
\left(h^{-1}\right)^{\prime}(v)=((n+1) F(v))^{-(1 /(n+1))},
$$

and

$$
\left(h^{-1}\right)^{\prime \prime}(v)=-f_{1}(v)((n+1) F(v))^{-(n+2 /(n+1))} .
$$

Hence $h$ is convex and strictly increasing. We see that

$$
\begin{aligned}
& K^{n}\left(\frac{1}{\left(h^{-1}\right)^{\prime}(v)^{n}}-|z|^{2} \frac{\left(h^{-1}\right)^{\prime \prime}(v)}{\left(h^{-1}\right)^{\prime}(v)^{n+2}}\right) \\
& =K^{n}\left(((n+1) F(v))^{(n /(n+1))}+|z|^{2} f_{1}(v)\right) \\
& =K^{n}\left(\frac{((n+1) F(v))^{(n /(n+1))}}{f_{1}(v)}+|z|^{2}\right) f_{1}(v) .
\end{aligned}
$$

We shall now show that

$$
\frac{((n+1) F(v(z)))^{(n /(n+1))}}{f_{1}(v(z))}+|z|^{2}
$$

is a smooth function which is bounded. It is smooth because it is the sum of a smooth function and a function which is a composition of smooth functions. Since $f_{1}$ is strictly positive we have to show that

$$
\frac{((n+1) F(v))^{(n /(n+1))}}{f_{1}(v)}
$$

is bounded for large values of $v$. We have

$$
\frac{\mathrm{d}}{\mathrm{d} v}\left(\frac{1}{\left(h^{-1}\right)^{\prime}(v)}\right)=\frac{f_{1}(v)}{((n+1) F(v))^{(n /(n+1))}}
$$

and this quantity must be larger than 1 for large $v$. Assume that

$$
\frac{\mathrm{d}}{\mathrm{d} v}\left(\frac{1}{\left(h^{-1}\right)^{\prime}(v)}\right)=\frac{\mathrm{d}}{\mathrm{d} v}\left(((n+1) F(v))^{(1 /(n+1))}\right)<1
$$


for large $v$. This implies that

$$
((n+1) F(v))^{(1 /(n+1))}<v+C
$$

for large $v$ which contradicts the integrability of $((n+1) F(v))^{-1 /(n+1)}$. Hence

$$
\frac{\mathrm{d}}{\mathrm{d} v}\left(\frac{1}{\left(h^{-1}\right)^{\prime}(v)}\right) \geq 1
$$

and

$$
\frac{((n+1) F(v))^{(n /(n+1))}}{f_{1}(v)} \leq 1
$$

for large $v$. Now choose $K$ so that

$$
K^{n}\left(\frac{1}{\left(h^{-1}\right)^{\prime}(v)^{n}}-|z|^{2} \frac{\left(h^{-1}\right)^{\prime \prime}(v)}{\left(h^{-1}\right)^{\prime}(v)^{n+2}}\right) \leq k(|z|) f_{1}(v) .
$$

By Lemma 1.3 we have $u_{N} \leq v$. Hence $u$ exists and what remains is to show that it is smooth. We take $R^{\prime}<R$ and shall prove that the norms $\left\|u_{N}\right\|_{2, \alpha}$ is uniformly bounded. We then use Schauder theory to conclude that $u \in C^{\infty}\left(B_{R^{\prime}}(0)\right)$ and since $R^{\prime}$ is arbitrary we have $u \in C^{\infty}\left(B_{R}(0)\right)$.

In order to use Propositions 2.2 and 2.3 we need to modify $u_{N}$. Let $\widetilde{R}=$ $\left(R+R^{\prime}\right) / 2$. Then $B_{R^{\prime}}(0) \Subset B_{\widetilde{R}}(0) \Subset B_{R}(0)$. Since $u_{N}$ is radial there are constants $\alpha_{N}=\left.u_{N}\right|_{\partial B_{\widetilde{R}}(0)}$ which are uniformly bounded because $u_{N} \leq v$ for all $N$. Put $\widetilde{u}_{N}=u_{N}-\alpha_{N}$ and $g_{N}(t)=f_{1}\left(t+\alpha_{N}\right)$. Note that $\widetilde{u}_{N} \equiv 0$ on $\partial B_{\widetilde{R}}(0)$ and that

$$
g_{N}\left(\widetilde{u}_{N}\right)=f_{1}\left(u_{N}\right) \leq f_{1}\left(\sup \left(v(z) ; z \in B_{\widetilde{R}}(0)\right)\right)
$$

in $B_{\widetilde{R}}(0)$. Also

$$
\operatorname{det}\left(\frac{\partial^{2} \widetilde{u}_{N}}{\partial z_{j} \partial \bar{z}_{k}}\right)=\operatorname{det}\left(\frac{\partial^{2} u_{N}}{\partial z_{j} \partial \bar{z}_{k}}\right)=k(|z|) f_{1}\left(u_{N}\right)=k(|z|) g_{N}\left(\widetilde{u}_{N}\right) .
$$

We begin by estimating the first derivatives of $u_{N}$. This is the same as estimating first derivatives of $\widetilde{u}_{N}$. For this we shall use Proposition 2.2. Since $\widetilde{u}_{N}$ is radial the function $U_{N}(|z|)=\widetilde{u}_{N}(z)$ is increasing. It follows that the constant $M$ in Proposition 2.2 is zero for all $N$. Since $k$ is smooth the constant $C$ in Proposition 2.2 is bounded. We see that

$$
\begin{aligned}
& \sup \left(\left|\frac{\partial u_{N}}{\partial x_{l}}(z)\right| ; z \in B_{R^{\prime}}(0)\right) \\
& \leq \frac{2 \sup \left(\left|v(z)-u_{1}(z)\right| ; z \in B_{R^{\prime}}(0)\right)+8 C \widetilde{R}^{3}}{\widetilde{R}-R^{\prime}}+4 C \widetilde{R}^{2}
\end{aligned}
$$

for all $N$ and $l=1, \ldots, 2 n$. We proceed to the estimate of the second derivatives and to get these estimates we are going to use that the solutions are radial. The Monge-Ampère equation can therefore be written as an ordinary differential equation. Using Lemma 1.2 we see that $\widetilde{u}_{N+1} \leq \widetilde{u}_{N}$ in $B_{\widetilde{R}}(0)$. This lets us conclude that $U_{N}^{\prime}(\widetilde{R}) \leq U_{N+1}^{\prime}(\widetilde{R})$. In fact this is not only true for the point $\widetilde{R}$. One can easily 
repeat the argument for balls with arbitrary radius and get $U_{N}^{\prime}(r) \leq U_{N+1}^{\prime}(r)$ for $0 \leq r<R$.

For radial functions one can write

$$
\operatorname{det}\left(\frac{\partial^{2} u}{\partial z_{j} \partial \bar{z}_{k}}\right)=2^{-(n+1)}\left(\frac{U^{\prime}(|z|)^{n}}{|z|^{n}}+\frac{U^{\prime}(|z|)^{n-1} U^{\prime \prime}(|z|)}{|z|^{n-1}}\right)
$$

where $U(|z|)=u(z)$. Therefore the solutions $U_{N}$ satisfy the equations

$$
\frac{U_{N}^{\prime \prime}\left(U_{N}^{\prime}\right)^{n-1}}{r^{n-1}}+\frac{\left(U_{N}^{\prime}\right)^{n}}{r^{n}}=2^{n+1} f_{1}\left(U_{N}\right) k(r)
$$

where $U_{N}(R)=N$ and $U_{N}^{\prime}(0)=0$. If we rearrange these equations we find that

$$
U_{N}^{\prime \prime}=\frac{2^{n+1} r^{n-1} f_{1}\left(U_{N}\right) k(r)}{\left(U_{N}^{\prime}\right)^{n-1}}-\frac{U_{N}^{\prime}}{r} .
$$

We are going to use these equations to get a uniform estimate of the second derivative of the solutions. First note that if we let $r$ tend to 0 we get

$$
U_{N}^{\prime \prime}(0)=\frac{2^{n+1} f_{1}\left(U_{N}(0)\right) k(0)}{\left(U_{N}^{\prime \prime}(0)\right)^{n-1}}-U_{N}^{\prime \prime}(0) .
$$

After a rearrangement we get

$$
U_{N}^{\prime \prime}(0)=2\left(f_{1}\left(U_{N}(0)\right) k(0)\right)^{1 / n}
$$

as expected. Inspecting the right-hand side of the Eq. (4) we see that $\sup \left(\left|U_{N}^{\prime \prime}(r)\right| ; 0 \leq r \leq \widetilde{R}\right)<M_{N}<\infty$ for each $N$. In principle we could have $\lim _{N \rightarrow \infty}=M_{N}=\infty$ since $U_{N}^{\prime}$ tends to 0 as $r$ tends to 0 . Therefore one has to eliminate the possibility that the quantities

$$
\sup \left(\frac{r^{n-1}}{U_{N}^{\prime}(r)^{n-1}} ; 0 \leq r \leq \widetilde{R}\right)
$$

grows uncontrollably in $N$. However we have already seen that $U_{N}^{\prime}(r) \leq U_{N+1}^{\prime}(r)$ and hence

$$
\sup \left(\frac{r^{n-1}}{U_{N}^{\prime}(r)^{n-1}} ; 0 \leq r \leq \widetilde{R}\right) \leq \sup \left(\frac{r^{n-1}}{U_{1}^{\prime}(r)^{n-1}} ; 0 \leq r \leq \widetilde{R}\right)<\infty .
$$

We get a uniform estimate of second derivatives on $B_{\widetilde{R}}(0)$ and an application of Proposition 2.3 gives a uniform Hölder estimate on the second derivative and that finishes the proof. 


\section{Blow-up estimates}

In order to estimate the blow-up rate of a solution to Problem (1) we prove a proposition on the blow-up rate of solutions to Problem (3).

Proposition 3.1. Assume that $f_{1} \in C^{\infty}(\mathbb{R})$ satisfies assumptions (A) and (C). Assume that $c_{1}, c_{2}$ is strictly positive numbers and that the function $k:[0, R] \rightarrow$ $\left[c_{1}, c_{2}\right]$ is a smooth function such that $k^{(2 l+1)}(0)=0$ for all $l \in \mathbb{N}$. Then a radial solution of Problem (3) meets the estimate

$$
\lim _{|z| \rightarrow R} \frac{\Psi_{n}(v(z))}{R-|z|}=2 R^{(n-1 /(n+1))} k(R)^{(1 /(n+1))} .
$$

Remark 3.2. The same proof technique was presented in [10] for a similar problem involving the real Monge-Ampère operator.

Proof. The existence of $v(|z|)=v(r)$ follows from Proposition 2.1. If we apply the Monge-Ampère operator to the radial function $v$ and perform the substitution $x=r^{m}$ with $m=2 n /(n+1)$ as above we obtain an equality which after a multiplication by $x^{s} g^{\prime}(x)$ can be written as

$$
\frac{\mathrm{d}}{\mathrm{d} x}\left(x^{s} g^{\prime}(x)^{s}\right)=2^{s} \frac{s}{m^{s}} k_{1}(x) x^{s} F^{\prime}(g(x))
$$

where $g(x)=v(r), s=n+1, k_{1}(x)=k(r)$ and $F(t)$ is the primitive function of $f_{1}(t)$ which is zero at the origin. Let us outline the calculation that leads to Eq. (5). Since $v$ is radial we have

$$
\operatorname{det}\left(\frac{\partial^{2} v}{\partial z_{j} \partial \bar{z}_{k}}(r)\right)=\frac{1}{2^{s} r^{s-2}}\left(v^{\prime \prime}(r) v^{\prime}(r)^{s-2}+\frac{v^{\prime}(r)^{s-1}}{r}\right) .
$$

Also since $v(r)=g\left(r^{m}\right)$ we see that $v^{\prime}(r)=m r^{m-1} g^{\prime}\left(r^{m}\right)$ and $v^{\prime \prime}(r)=m(m-$ 1) $r^{m-2} g^{\prime}\left(r^{m}\right)+m^{2} r^{2 m-2} g^{\prime \prime}\left(r^{m}\right)$. Therefore

$$
\begin{aligned}
& \operatorname{det}\left(\frac{\partial^{2} v}{\partial z_{j} \partial \bar{z}_{k}}(r)\right) \\
& =\frac{m^{s}}{2^{s}}\left(r^{(m-2)(s-1)} g^{\prime}\left(r^{m}\right)^{s-1}+r^{(m-2) s+2} g^{\prime \prime}\left(r^{m}\right) g^{\prime}\left(r^{m}\right)^{s-2}\right) \\
& =\frac{m^{s}}{2^{s}} r^{(m-2) s+2}\left(\frac{g^{\prime}\left(r^{m}\right)^{s-1}}{r^{m}}+g^{\prime \prime}\left(r^{m}\right) g^{\prime}\left(r^{m}\right)^{s-2}\right) \\
& =\frac{m^{s}}{2^{s}}\left(\frac{g^{\prime}\left(r^{m}\right)^{s-1}}{r^{m}}+g^{\prime \prime}\left(r^{m}\right) g^{\prime}\left(r^{m}\right)^{s-2}\right) .
\end{aligned}
$$

We also have

$$
\begin{aligned}
\frac{\mathrm{d}}{\mathrm{d} x}\left(x^{s} g^{\prime}(x)^{s}\right) & =s x^{s-1} g^{\prime}(x)^{s}+s x^{s} g^{\prime \prime}(x) g^{\prime}(x)^{s-1} \\
& =s x^{s} g^{\prime}(x)\left(\frac{g^{\prime}(x)^{s-1}}{x}+g^{\prime \prime}(x) g^{\prime}(x)^{s-2}\right)
\end{aligned}
$$

and this yields Eq. (5). 
Let $0 \leq t_{1} \leq t_{2} \leq t \leq R^{m}$ where we shall choose $t_{1}$ and $t_{2}$ shortly. When we integrate equation ( $\dagger$ ) from 0 to $t \leq R^{m}$ we obtain

$$
\begin{aligned}
t^{s} g^{\prime}(t)^{s}= & 2^{s} \frac{s}{m^{s}} \int_{0}^{t} k_{1}(x) x^{s} F^{\prime}(g(x)) \mathrm{d} x \\
= & 2^{s} \frac{s}{m^{s}}\left(\int_{0}^{t_{1}} k_{1}(x) x^{s} F^{\prime}(g(x)) \mathrm{d} x+\int_{t_{1}}^{t} k_{1}(x) x^{s} F^{\prime}(g(x)) \mathrm{d} x\right) \\
= & 2^{s} \frac{s}{m^{s}}\left(k_{1}\left(\eta_{1}\right) \eta_{1}^{s}\left(F\left(g\left(t_{1}\right)\right)-F(g(0))\right)\right. \\
& \left.+k_{1}\left(\eta_{2}\right) \eta_{2}^{s}\left(F(g(t))-F\left(g\left(t_{1}\right)\right)\right)\right)
\end{aligned}
$$

for some $\eta_{1} \in\left[0, t_{1}\right]$ and $\eta_{2} \in\left[t_{1}, t\right]$. Fix $\varepsilon>0$ and choose $t_{1} \in\left[0, R^{m}\right]$ such that $\left(R^{m} / t_{1}\right)^{s}<1+\varepsilon$ and $\left|k_{1}\left(R^{m}\right) R^{m s}-k_{1}(\eta) \eta^{s}\right|<\varepsilon$ for $\eta \in\left[t_{1}, R^{m}\right]$. Since $\lim _{t \rightarrow R^{m}} F(g(t))=\infty$ it is possible to choose $t_{2} \geq t_{1}$ such that

$$
\begin{aligned}
& \left|k_{1}\left(\eta_{1}\right) \eta_{1}^{s}\left(F\left(g\left(t_{1}\right)\right)-F(g(0))\right)-\left(k_{1}\left(R^{m}\right) R^{m s}-\varepsilon\right) F\left(g\left(t_{1}\right)\right)\right| \\
& \quad \leq \varepsilon\left(k_{1}\left(R^{m}\right) R^{m s}+\varepsilon\right) F(g(t))
\end{aligned}
$$

when $t \in\left[t_{2}, R^{m}\right]$. Hence we have

$$
\begin{aligned}
g^{\prime}(t)^{s} & \leq 2^{s} \frac{s}{m^{s}} \frac{1}{t^{s}}(1+\varepsilon)\left(k_{1}\left(R^{m}\right) R^{m s}+\varepsilon\right) F(g(t)) \\
& \leq 2^{s} \frac{s}{m^{s}}(1+\varepsilon)\left(k_{1}\left(R^{m}\right)(1+\varepsilon)^{s}+\frac{\text { varepsilon }(1+\varepsilon)}{R^{m s}}\right) F(g(t))
\end{aligned}
$$

for $t \in\left[t_{2}, R^{m}\right]$ and we obtain

$$
\begin{aligned}
\Psi_{n}(v(r)) & =\int_{r^{m}}^{R^{m}}(s F(g(x)))^{-1 / s} g^{\prime}(x) \mathrm{d} x \\
& \leq \frac{2}{m} \int_{r^{m}}^{R^{m}}\left((1+\varepsilon)\left(k_{1}\left(R^{m}\right)(1+\varepsilon)^{s}+\frac{\varepsilon(1+\varepsilon)}{R^{m s}}\right)\right)^{1 / s} \mathrm{~d} x \\
& =\frac{2}{m}\left((1+\varepsilon)\left(k_{1}\left(R^{m}\right)(1+\varepsilon)^{s}+\frac{\varepsilon(1+\varepsilon)}{R^{m s}}\right)\right)^{1 / s}\left(R^{m}-r^{m}\right)
\end{aligned}
$$

where $r^{m} \geq t_{2}$. Since $\lim _{r \rightarrow R}\left(R^{m}-r^{m} /(R-r)\right)=m R^{m-1}$ we have

$$
\lim _{r \rightarrow R} \frac{\Psi_{n}(v(r))}{R-r} \leq 2\left((1+\varepsilon)\left(k(R)(1+\varepsilon)^{n+1}+\frac{\varepsilon(1+\varepsilon)}{R^{2 n}}\right)\right)^{(1 /(n+1))} R^{(n-1 /(n+1))} .
$$

To prove the converse inequality, we use Eq. (5) again, but this time we integrate the equality from $t_{0}$ to $t, 0<t_{0}<t<R^{m}$ and get

$$
t^{s} g^{\prime}(t)^{s}-t_{0}^{s} g^{\prime}\left(t_{0}\right)^{s}=2^{s} \frac{s}{m^{s}} \int_{t_{0}}^{t} x^{s} k_{1}(x) F^{\prime}(g(x)) \mathrm{d} x .
$$


Dividing by $t^{s}$ and adding $(1-1) k_{1}(x) F^{\prime}(g(x))$ to the integrand gives

$$
\begin{aligned}
g^{\prime}(t)^{s}= & \left(\frac{t_{0}}{t}\right)^{s} g^{\prime}\left(t_{0}\right)^{s}+2^{s} \frac{s}{m^{s}} \int_{t_{0}}^{t} k_{1}(x) F^{\prime}(g(x)) \mathrm{d} x \\
& +2^{s} \frac{s}{m^{s}} \int_{t_{0}}^{t}\left(\left(\frac{x}{t}\right)^{s}-1\right) k_{1}(x) F^{\prime}(g(x)) \mathrm{d} x .
\end{aligned}
$$

We have the estimates

$$
\int_{t_{0}}^{t} k_{1}(x) F^{\prime}(g(x)) \mathrm{d} x \geq \inf \left(k_{1}(\xi) ; \xi \in\left[t_{0}, t\right]\right)\left(F(g(t))-F\left(g\left(t_{0}\right)\right)\right)
$$

and

$$
\begin{aligned}
& \left|\int_{t_{0}}^{t}\left(\left(\frac{x}{t}\right)^{s}-1\right) k_{1}(x) F^{\prime}(g(x)) \mathrm{d} x\right| \\
& \quad \leq \sup \left(\left|\left(\left(\frac{\xi}{t}\right)^{s}-1\right) k_{1}(\xi)\right| ; \xi \in\left[t_{0}, t\right]\right)\left(F(g(t))-F\left(g\left(t_{0}\right)\right)\right) .
\end{aligned}
$$

Choose $t_{0} \in\left(0, R^{m}\right)$ such that

$$
\sup \left(\left|\left(\left(\frac{\xi}{t}\right)^{s}-1\right) k_{1}(\xi)\right| ; \quad \xi \in\left[t_{0}, t\right]\right)<\varepsilon
$$

and

$$
\inf \left(k_{1}(\xi) ; \xi \in\left[t_{0}, t\right]\right)>k_{1}\left(R^{m}\right)-\varepsilon .
$$

Since $\lim _{t \rightarrow R^{m}} F(g(t))=\infty$ it is possible to choose $t_{1}>t_{0}$ such that $F\left(g\left(t_{0}\right)\right)<$ $\varepsilon F(g(t))$ when $t \in\left[t_{1}, R^{m}\right]$. For these $t$ we have, since $g^{\prime}\left(t_{0}\right)$ is positive,

$$
g^{\prime}(t)^{s} \geq 2^{s} \frac{s}{m^{s}} F(g(t))\left(k_{1}\left(R^{m}\right)-2 \varepsilon\right)(1-\varepsilon) .
$$

Hence

$$
\lim _{r \rightarrow R} \frac{\Psi_{n}(v(r))}{R-r} \geq 2((k(R)-2 \varepsilon)(1-\varepsilon))^{(1 /(n+1))} R^{(n-1 /(n+1))}
$$

and if we let $\varepsilon$ tend to zero the proposition follows.

Remark 3.3. In proving the upper bound for $\lim _{r \rightarrow R}\left(\Psi_{n}(v(r)) / R-r\right)$ we could have integrated Eq. (5) by parts and obtained

$$
t^{s} g^{\prime}(t)^{s}=2^{s} \frac{s}{m^{s}} k_{1}(t) t^{s} F(g(t))-2^{s} \frac{s}{m^{s}} \int_{0}^{t} \frac{\mathrm{d}}{\mathrm{d} x}\left(k_{1}(x) x^{s}\right) F(g(x)) \mathrm{d} x .
$$

If $\mathrm{d} / \mathrm{d} x\left(k_{1}(x) x^{s}\right) \geq 0$, which is the case when $k_{1}$ is constant, we could ignore the last integral since it is positive and get easier calculations. 
We are now ready to estimate the boundary blow-up rate of the solution $u$ to Problem (1), our goal being an estimate in terms of the distance from $z$ to the boundary of $\Omega$ and the product of the eigenvalues of the Levi form. First we show an inequality and then we refine the argument to get an equality. We begin by deriving an upper bound which is easy. Take $z_{0} \in \partial \Omega$. Since $\Omega$ has smooth boundary there exists a ball $B_{R}\left(\tilde{z}_{0}\right)$ with radius $R$ and center $\tilde{z}_{0}$ such that $B_{R}\left(\tilde{z}_{0}\right) \subseteq \Omega$ and $z_{0} \in \partial B_{R}\left(\tilde{z}_{0}\right)$. Now, for $0 \leq \varepsilon<R$, solve

$$
\left\{\begin{array}{l}
\operatorname{det}\left(\frac{\partial^{2} v_{\varepsilon}}{\partial z_{j} \partial \bar{z}_{k}}\right)=c_{1} f_{1}\left(v_{\varepsilon}(z)\right) \text { in } B_{R-\varepsilon}\left(\tilde{z}_{0}\right) \\
\lim _{|z| \rightarrow R-\varepsilon} v_{\varepsilon}(z)=\infty
\end{array}\right.
$$

By Lemma 1.3 we have $u \leq v_{\varepsilon}$ in $B_{R-\varepsilon}\left(\tilde{z}_{0}\right)$ for $0<\varepsilon<R$ and since $v_{0}(z)=$ $\lim _{\varepsilon \rightarrow 0} v_{\varepsilon}(z)$ in $B_{R}\left(\tilde{z}_{0}\right)$ it follows that $u \leq v$ in $B_{r}\left(\tilde{z}_{0}\right)$. Hence $\Psi_{n}\left(u\left(\left|z-\tilde{z}_{0}\right|\right)\right) \geq$ $\Psi_{n}\left(v_{0}\left(\left|z-\tilde{z}_{0}\right|\right)\right)$ and using Proposition 3.1 we see that

$$
\lim _{r \rightarrow R} \frac{\Psi_{n}\left(u\left(\tilde{z}_{0}+(r / R)\left(z_{0}-\tilde{z}_{0}\right)\right)\right)}{R-r} \geq 2 c_{1}^{(1 /(n+1))} R^{(n-1 /(n+1))} .
$$

The lower bound is a little trickier. If $\Omega$ were strongly convex we could compare $u$ with a solution of a related radial problem in a ball containing $\Omega$, which touches $\partial \Omega$ at a single boundary point $z_{0} \in \partial \Omega$. Modulo technical arguments this idea gives a good lower estimate of the boundary blow-up rate of $u$ at $z_{0}$. It is obvious that the above technique cannot be used if $\Omega$ is merely strongly pseudoconvex. Here a second idea is needed, namely given $z_{0} \in \partial \Omega$ where $u$ is to be estimated from below, we use a lemma of Narasimhan [9] to map a neighborhood of $z_{0}$ biholomorphically onto a strongly convex domain and thus obtain a local transformation of the problem to a situation we can handle.

The local character of the problem introduces an new obstacle too, since we do not know that the transformed version of $u$, let us call it $\tilde{u}$, is big enough at all boundary points of the new domain: Boundary blow-up occurs only on a part of the boundary containing the image point of $z_{0}$. This problem is overcome by constructing a very bad lower bound for $\tilde{u}$ which however is good enough at the boundary point in question. When comparing with a radial solution of the related problem in a ball containing the transformed neighborhood of $z_{0}$, we push this radial solution below $\tilde{u}$ on the problematic part of the boundary by subtracting an affine function.

We need the following lemma of Narasimhan [9].

Lemma 3.4. Let $\Omega \Subset \mathbb{C}^{n}$ be a domain with a $C^{2}$ boundary. Let $z_{0} \in \partial \Omega$ be a point of strong pseudoconvexity. Then there exists a neighborhood $Z \subseteq \mathbb{C}^{n}$ of $z_{0}$ and a biholomorphic mapping $\Phi$ on $Z$ such that $W=\Phi(Z \cap \Omega)$ is strongly convex.

We will need the form of the biholomorphism $\Phi$. It is known that $\Omega$ has a defining function $\rho$ with the property that there exists $C>0$ such that

$$
\sum_{j, k=1}^{n} \frac{\partial^{2} \rho}{\partial z_{j} \partial \bar{z}_{k}}\left(z_{0}\right) z_{j} \bar{z}_{k} \geq C|z|^{2}
$$


for all $z_{0} \in \partial \Omega$ and all $z \in \mathbb{C}^{n}$. The local biholomorphism at $z_{0} \in \partial \Omega$ can, after a translation taking $z_{0}$ to zero and a rotation taking the exterior normal at $z_{0}$ to $(1,0, \ldots, 0)$, be written as

$$
w_{1}=\Phi_{1}(z)=z_{1}+(1 / 2)\left(\sum_{j, k=1}^{n} \frac{\partial^{2} \rho}{\partial z_{j} \partial z_{k}}\left(z_{0}\right) z_{j} z_{k}\right), \quad w_{j}=\Phi_{j}(z)=z_{j}
$$

for $j=2, \ldots, n$. Now define $\tilde{u}: W \rightarrow \mathbb{R}$ as $\tilde{u}(w)=u\left(\Phi^{-1}(w)\right)$. The MongeAmpère operator transforms as

$$
\operatorname{det}\left(\frac{\partial^{2} \tilde{u}}{\partial w_{j} \partial \bar{w}_{k}}(w)\right)=\left|\operatorname{det}\left(\Phi^{-1}\right)^{\prime}(w)\right|^{2} \operatorname{det}\left(\frac{\partial^{2} u}{\partial z_{j} \partial \bar{z}_{k}}\left(\Phi^{-1}(w)\right)\right)
$$

under holomorphic coordinate changes. Since $W$ is strongly convex there is a ball $B_{R^{\prime}}\left(\tilde{w}_{0}\right)$ with radius $R^{\prime}$ and center $\tilde{w}_{0}$ having the properties that $W \subseteq B_{R^{\prime}}\left(\tilde{w}_{0}\right)$ and $\partial B_{R^{\prime}}\left(\tilde{w}_{0}\right) \cap \partial W=\left\{w_{0}\right\}$, where $w_{0}=\Phi\left(z_{0}\right)$. Let $\eta>0$ and if we shrink $W$ if necessary we can assume that $\left.|| \operatorname{det}\left(\Phi^{-1}\right)^{\prime}(w)\right|^{2}-\left|\operatorname{det}\left(\Phi^{-1}\right)^{\prime}\left(w_{0}\right)\right|^{2} \mid \leq \eta$ on $W$. Take a smooth $g$ which satisfies

$$
g(s) \geq \sup \left(c_{2}\left|\operatorname{det}\left(\Phi^{-1}\right)^{\prime}(w)\right|^{2} ;\left\{w ;\left|w-\tilde{w}_{0}\right|=s\right\}\right)
$$

and $g\left(R^{\prime}\right)=c_{2}\left(\left|\operatorname{det}\left(\Phi^{-1}\right)^{\prime}\left(w_{0}\right)\right|^{2}+\eta\right)=c_{2}(1+\eta)$. Since we want to study Problem (3) in $B_{R^{\prime}}\left(\tilde{w}_{0}\right)$ and use Proposition 3.1 we need to extend $g$ in such a way that the proposition is still applicable if $\tilde{w}_{0} \notin W$. Abusing notation let us call this extension $g$. Take $\varepsilon>0$. We extend $g$ so that Proposition 3.1 can be applied in $B_{R^{\prime}+\varepsilon}\left(\tilde{w}_{0}\right)$ and solve Problem (3) with right-hand side $g\left(\left|w-\tilde{w}_{0}\right|\right) f_{1}(t)$ in $B_{R^{\prime}+\varepsilon}\left(\tilde{w}_{0}\right)$. Let us call the solution $\widetilde{v}_{\varepsilon}$. Put $\widetilde{v}(z)=\lim _{\varepsilon \rightarrow 0+} \widetilde{v}_{\varepsilon}(z)$. The function $\widetilde{v}$ is a smooth solution to Problem (3) in $B_{R^{\prime}}\left(\tilde{w}_{0}\right)$ with right-hand side $g\left(\left|w-\tilde{w}_{0}\right|\right) f_{1}(t)$. Since

$$
\begin{aligned}
\operatorname{det}\left(\frac{\partial^{2} \tilde{u}}{\partial w_{j} \partial \bar{w}_{k}}(w)\right) & =\left|\operatorname{det}\left(\Phi^{-1}\right)^{\prime}(w)\right|^{2} \operatorname{det}\left(\frac{\partial^{2} u}{\partial z_{j} \partial \bar{z}_{k}}\left(\Phi^{-1}(w)\right)\right) \\
& =\left|\operatorname{det}\left(\Phi^{-1}\right)^{\prime}(w)\right|^{2} f\left(\Phi^{-1}(w), \tilde{u}(w)\right)
\end{aligned}
$$

and

$$
\begin{aligned}
\operatorname{det}\left(\frac{\partial^{2} \tilde{v}_{\varepsilon}}{\partial w_{j} \partial \bar{w}_{k}}(w)\right) & =g\left(\left|w-\tilde{w}_{0}\right|\right) f_{1}\left(\tilde{v}_{\varepsilon}(w)\right) \\
& \geq c_{2}\left|\operatorname{det}\left(\Phi^{-1}\right)^{\prime}(w)\right|^{2} f_{1}\left(\tilde{v}_{\varepsilon}(w)\right) \\
& \geq\left|\operatorname{det}\left(\Phi^{-1}\right)^{\prime}(w)\right|^{2} f\left(\Phi^{-1}(w), \tilde{v}_{\varepsilon}(w)\right)
\end{aligned}
$$

in $W$ we could conclude that $\tilde{v_{\varepsilon}} \leq \tilde{u}$ in $W$ if we knew that $\tilde{v_{\varepsilon}} \leq \tilde{u}$ on $\partial W$. This would imply that $\widetilde{v} \leq \widetilde{u}$ in $W$. To handle this we will make use of the function Re $w_{1}$. Let $\widetilde{W}=\partial W \backslash \Phi(\partial \Omega \cap Z)$. By Lemma 1.3 we have $\tilde{v}_{\varepsilon} \leq \tilde{v}$ in $B_{R^{\prime}}\left(\tilde{w}_{0}\right)$. Choose $\alpha \in \mathbb{R}$ such that

$$
\sup \left(\tilde{v}(w)+\alpha \operatorname{Re} w_{1} ; w \in \widetilde{W}\right) \leq \inf (\tilde{u}(w) ; w \in \widetilde{W}) .
$$


Since $\tilde{v}_{\varepsilon}+\alpha \operatorname{Re} w_{1} \leq \tilde{v}+\alpha \operatorname{Re} w_{1} \leq \tilde{u}$ on $\partial W$ we use Lemma 1.3 to conclude that $\tilde{v}_{\varepsilon}+\alpha \operatorname{Re} w_{1} \leq \tilde{u}$ in $W$ and letting $\varepsilon$ tend to zero we see that $\tilde{v}+\alpha \operatorname{Re} w_{1} \leq \tilde{u}$ in $W$. We also have

$$
\begin{aligned}
\Psi_{n}\left(\tilde{v}(w)+\alpha \operatorname{Re} w_{1}\right) & =\Psi_{n}(\tilde{v}(w))+\alpha \operatorname{Re} w_{1} \Psi_{n}^{\prime}(\xi) \\
& =\Psi_{n}(\tilde{v}(w))+\alpha \operatorname{Re} w_{1}((n+1) F(\xi))^{-(1 /(n+1))}
\end{aligned}
$$

where $\xi \in\left[\tilde{v}(w)+\alpha \operatorname{Re} w_{1}, \tilde{v}(w)\right]$. Hence we have

$$
\begin{aligned}
& \lim _{r \rightarrow R^{\prime}} \frac{\Psi_{n}\left(\tilde{u}\left(\tilde{w}_{0}+\left(r / R^{\prime}\right)\left(w_{0}-\tilde{w}_{0}\right)\right)\right)}{R^{\prime}-r} \\
& \leq \lim _{r \rightarrow R^{\prime}} \frac{\Psi_{n}\left(\tilde{v}\left(\tilde{w}_{0}+\left(r / R^{\prime}\right)\left(w_{0}-\tilde{w}_{0}\right)\right)+\alpha\left(R^{\prime}-r\right)\right)}{R^{\prime}-r} \\
& =\lim _{r \rightarrow R^{\prime}} \frac{\Psi_{n}\left(\tilde{v}\left(\tilde{w}_{0}+\left(r / R^{\prime}\right)\left(w_{0}-\tilde{w}_{0}\right)\right)+\alpha\left(R^{\prime}-r\right)((n+1) F(\xi))^{-(1 /(n+1))}\right.}{R^{\prime}-r} \\
& =\lim _{r \rightarrow R^{\prime}} \frac{\Psi_{n}\left(\tilde{v}\left(\tilde{w}_{0}+\left(r / R^{\prime}\right)\left(w_{0}-\tilde{w}_{0}\right)\right)\right.}{R^{\prime}-r}+\lim _{r \rightarrow R^{\prime}} \alpha((n+1) F(\xi))^{-(1 /(n+1))} \\
& \left.\quad=\lim _{r \rightarrow R^{\prime}} \frac{\Psi_{n}\left(\tilde{v}\left(\tilde{w}_{0}+\left(r / R^{\prime}\right)\left(w_{0}-\tilde{w}_{0}\right)\right)\right.}{R^{\prime}-r} R^{\prime}\right)^{(1 /(n+1))} R^{\prime(n-1 /(n+1))} \\
& \leq 2 c_{2}^{(1 /(n+1))}(1+\eta)^{(1 /(n+1))} R^{\prime(n-1 /(n+1))}
\end{aligned}
$$

by Proposition 3.1. Note that we are measuring the distance between $\Phi(z)$ and the boundary of $W$ and not between $z$ and the boundary of $\Omega$. This is easily handled if we put $\hat{z}_{0}=\Phi^{-1}\left(\tilde{w}_{0}\right), \widehat{R}=\left|\hat{z}_{0}\right|$ and observe the following

$$
\begin{aligned}
\lim _{r \rightarrow \widehat{R}} \frac{\Psi_{n}\left(u\left(\hat{z}_{0}\right)+(r / \widehat{R})\left(z_{0}-\hat{z}_{0}\right)\right)}{\widehat{R}-r} \\
\quad=\lim _{r \rightarrow \widehat{R}} \frac{\Psi_{n}\left(u\left(\hat{z}_{0}\right)+(r / \widehat{R})\left(z_{0}-\hat{z}_{0}\right)\right)}{\left.\mid r / \widehat{R})\left(z_{0}-\hat{z}_{0}\right)\right)-\Phi\left(z_{0}\right) \mid} \frac{\left|\Phi\left(\hat{z}_{0}+(r / \widehat{R})\left(z_{0}-\hat{z}_{0}\right)\right)-\Phi\left(z_{0}\right)\right|}{\widehat{R}-r} \\
\quad \leq 2 c_{2}^{(1 /(n+1))}(1+\eta)^{(1 /(n+1))} R^{\prime(n-1 /(n+1))} \lim _{\widehat{R} \rightarrow \widehat{R}} \frac{\left|\Phi\left(\hat{z}_{0}+(r / \widehat{R})\left(z_{0}-\hat{z}_{0}\right)\right)-\Phi\left(z_{0}\right)\right|}{\widehat{R}-r} \\
\quad=2 c_{2}^{(1 /(n+1))}(1+\eta)^{(1 /(n+1))} R^{\prime(n-1 /(n+1))} .
\end{aligned}
$$

Let $\eta$ tend to zero. We have proved a partial description of the blow-up rate of solutions when we approach a boundary point in the normal direction. We introduce some notation. For $z_{0} \in \partial \Omega$ let

$$
\mathcal{I}_{z_{0}}=\left\{R \in \mathbb{R} ; B_{R}(z) \subseteq \Omega \text { and } \partial B_{R}(z) \cap \partial \Omega=\left\{z_{0}\right\} \text { for some } z \in \Omega\right\}
$$

and

$\mathcal{I}_{z_{0}}^{\prime}=\left\{R \in \mathbb{R} ; W \subseteq B_{R}(z)\right.$ and $\partial B_{R}(z) \cap \partial W=\left\{\Phi\left(z_{0}\right)\right\}$ for some $\left.z \in \mathbb{C}^{n}\right\}$

where $\Phi$ and $W$ are described in Lemma 3.4. Put $R\left(z_{0}\right)=\sup \left(R ; R \in \mathcal{I}_{z_{0}}\right)$ and $R^{\prime}\left(z_{0}\right)=\inf \left(R ; R \in \mathcal{I}_{z_{0}}^{\prime}\right)$. The functions $R\left(z_{0}\right)$ and $R^{\prime}\left(z_{0}\right)$ are continuous. Using this we get the following. 
Proposition 3.5. Let $\Omega$ be a bounded, strongly pseudoconvex domain in $\mathbb{C}^{n}$ with smooth boundary. Let $f \in C^{\infty}(\bar{\Omega} \times \mathbb{R})$ be a strictly positive function which is increasing in the second variable and satisfies assumptions (A), (B) and (C). Then $u$, a solution to Problem (1), meets the estimate

$$
2 c_{1}^{(1 /(n+1))} R\left(z_{0}\right)^{(n-1 /(n+1))} \leq \lim _{z \rightarrow z_{0}} \frac{\Psi_{n}(u(z))}{d_{\Omega}(z)} \leq 2 c_{2}^{(1 /(n+1))} R^{\prime}\left(z_{0}\right)^{(n-1 /(n+1))},
$$

where $z_{0} \in \partial \Omega$.

Equipped with this we prove the following.

Proposition 3.6. Let $\Omega$ be a bounded, strongly pseudoconvex domain in $\mathbb{C}^{n}$ with smooth boundary. Let $f \in C^{\infty}(\bar{\Omega} \times \mathbb{R})$ be a strictly positive function which is increasing in the second variable and satisfies assumptions (A), (B) and (C). Then $u$, a solution to Problem (1), meets the estimate

$$
\begin{aligned}
2 h\left(z_{0}\right)^{(1 /(n+1))} R\left(z_{0}\right)^{(n-1 /(n+1))} & \leq \lim _{z \rightarrow z_{0}} \frac{\Psi_{n}(u(z))}{d_{\Omega}(z)} \\
& \leq 2 h\left(z_{0}\right)^{(1 /(n+1))} R^{\prime}\left(z_{0}\right)^{(n-1 /(n+1)),}
\end{aligned}
$$

where $z_{0} \in \partial \Omega$.

Proof. Fix $\varepsilon>0$ and $z_{0} \in \partial \Omega$. By assumption (A) there exists a constant $C \in \mathbb{R}$ such that

$$
(h(z)-\varepsilon) f_{1}(t) \leq f(z, t) \leq(h(z)+\varepsilon) f_{1}(t)
$$

for all $z \in \Omega$ if $t>C$. Using Proposition 3.5 we see that

$$
\lim _{z \rightarrow z_{0}} \frac{\Psi_{n}(u(z))}{d_{\Omega}(z)} \leq 2 c_{2}^{(1 /(n+1))} R^{\prime}\left(z_{0}\right)^{(n-1 /(n+1))} .
$$

Hence there exists $\delta>0$ such that

$$
\Psi_{n}(u(z)) \leq\left(2 c_{2}^{(1 /(n+1))} R^{\prime}\left(z_{0}\right)^{(n-1 /(n+1))}+\varepsilon\right) d_{\Omega}(z)
$$

if $\left|z-z_{0}\right|<\delta$. Since $\Psi_{n}$ is decreasing $\Psi_{n}^{-1}$ is and since $\lim _{t \rightarrow \infty} \Psi_{n}(t)=0$ there exists $\delta^{\prime} \leq \delta$ such that

$$
u(z) \geq \Psi_{n}^{-1}\left(\left(2 c_{2}^{(1 /(n+1))} R^{\prime}\left(z_{0}\right)^{(n-1 /(n+1))}+\varepsilon\right) d_{\Omega}(z)\right) \geq C
$$

if $\left|z-z_{0}\right|<\delta^{\prime}$. Now take $R \in \mathcal{I}_{z_{0}}$ and choose $\delta^{\prime \prime}<\delta^{\prime}$ such that

$$
\sup \left(\left|h(z)-h\left(z_{0}\right)\right| ; z \in B_{R}\left(\tilde{z}_{0}\right) \cap B_{\delta^{\prime \prime}}\left(z_{0}\right)\right) \leq \varepsilon .
$$

Here $B_{R}\left(\tilde{z}_{0}\right) \subseteq \Omega$ such that $\partial B_{R}\left(\tilde{z}_{0}\right) \cap \partial \Omega$. Let $g$ be a strictly positive smooth function which satisfies

$$
g(s) \leq \inf \left(h(z)-\varepsilon ;\left\{z \in B_{R}\left(\tilde{z}_{0}\right) \cap B_{\delta^{\prime \prime}}\left(z_{0}\right) ;\left|z-\tilde{z}_{0}\right|=s\right\}\right)
$$


for $s \in\left[R-\delta^{\prime \prime}, R\right]$ and $g(R)=h\left(z_{0}\right)-2 \varepsilon$. If $R-\delta^{\prime \prime}>0$ extend $g$ to $[0, R]$ in such a way that Proposition 3.1 still can be used. Abusing notation again call this extension $g$. We may assume, after a translation and rotation, that $z_{0}=0$ and the exterior normal at $z_{0}$ is $(1,0, \ldots, 0)$. Solve Problem (3) in a slightly smaller ball $B_{R-\varepsilon^{\prime}}\left(\tilde{z}_{0}\right)$ and call this solution $v_{\varepsilon^{\prime}}$. Put $v(z)=\lim _{\mathcal{\varepsilon}^{\prime} \rightarrow 0} v_{\varepsilon^{\prime}}(z)$, which exists since $v_{\mathcal{E}^{\prime}}(z) \leq v_{\varepsilon^{\prime \prime}}(z)$ when $\varepsilon^{\prime} \leq \varepsilon^{\prime \prime}$ and $v_{\mathcal{E}^{\prime}}(z) \geq \widetilde{v}(z)$ for any solution $\widetilde{v}$ of Problem (1) in $B_{R}\left(\widetilde{z}_{0}\right)$ with right-hand side $g\left(\left|z-\widetilde{z}_{0}\right|\right) f_{1}(t)$. Then $v$ is a smooth solution of Problem (1) in $B_{R}\left(\widetilde{z}_{0}\right)$ with right-hand side $g\left(\left|z-\widetilde{z}_{0}\right|\right) f_{1}(t)$. Now choose $\alpha \in \mathbb{R}$ such that

$$
\begin{aligned}
& \inf \left(v(z)-\alpha \operatorname{Re} z_{1} ; z \in B_{R}\left(\tilde{z}_{0}\right) \cap \partial B_{\delta^{\prime \prime}}\left(z_{0}\right)\right) \\
& \geq \sup \left(u(z) ; z \in B_{R}\left(\tilde{z}_{0}\right) \cap \partial B_{\delta^{\prime \prime}}\left(z_{0}\right)\right) .
\end{aligned}
$$

We have

$$
\begin{aligned}
\operatorname{det}\left(\frac{\partial^{2}\left(v_{\varepsilon^{\prime}}-\alpha \operatorname{Re} z_{1}\right)}{\partial z_{j} \partial \bar{z}_{k}}(z)\right) & =\operatorname{det}\left(\frac{\partial^{2} v_{\varepsilon^{\prime}}}{\partial z_{j} \partial \bar{z}_{k}}(z)\right)=g\left(\left|z-\tilde{z}_{0}\right|\right) f_{1}\left(v_{\mathcal{\varepsilon}^{\prime}}(z)\right) \\
& \leq(h(z)-\varepsilon) f_{1}\left(v_{\mathcal{\varepsilon}^{\prime}}(z)\right) \leq f\left(z, v_{\mathcal{\varepsilon}^{\prime}}(z)\right) \\
& \leq f\left(z, v_{\mathcal{\varepsilon}^{\prime}}(z)-\alpha \operatorname{Re} z_{1}\right)
\end{aligned}
$$

in $B_{R-\varepsilon^{\prime}}\left(\tilde{z}_{0}\right) \cap B_{\delta^{\prime \prime}}\left(z_{0}\right)$ and if we use Lemma 1.3 we see that $v_{\mathcal{E}^{\prime}}-\alpha \operatorname{Re} z_{1} \geq u$ in $B_{R-\varepsilon^{\prime}}\left(\tilde{z}_{0}\right) \cap B_{\delta^{\prime \prime}}\left(z_{0}\right)$. Letting $\varepsilon^{\prime}$ tend to zero we conclude that $v-\alpha \operatorname{Re} z_{1} \geq u$ in $B_{R}\left(\tilde{z}_{0}\right) \cap B_{\delta^{\prime \prime}}\left(z_{0}\right)$. Using Proposition 3.1 and noting that $g(R)=h\left(z_{0}\right)-2 \varepsilon$ we see that

$$
2\left(h\left(z_{0}\right)-2 \varepsilon\right)^{(1 /(n+1))} R^{(n-1 /(n+1))} \leq \lim _{z \rightarrow z_{0}} \frac{\Psi_{n}(u(z))}{d_{\Omega}(z)} .
$$

If we let $\varepsilon$ tend to zero and observe that $R \in \mathcal{I}_{z_{0}}$ was arbitrary we get

$$
2 h\left(z_{0}\right)^{(1 /(n+1))} R\left(z_{0}\right)^{(n-1 /(n+1))} \leq \lim _{z \rightarrow z_{0}} \frac{\Psi_{n}(u(z))}{d_{\Omega}(z)} .
$$

If we modify the proof of the lower bound in Proposition 3.5 slightly we finally arrive at

$$
\begin{aligned}
2 h\left(z_{0}\right)^{(1 /(n+1))} R\left(z_{0}\right)^{(n-1 /(n+1))} & \leq \lim _{z \rightarrow z_{0}} \frac{\Psi_{n}(u(z))}{d_{\Omega}(z)} \\
& \leq 2 h\left(z_{0}\right)^{(1 /(n+1))} R^{\prime}\left(z_{0}\right)^{(n-1 /(n+1))} .
\end{aligned}
$$

Using the idea of adding and subtracting affine functions we can refine the argument above and get the following improvement. Let us first introduce some notation.

Definition 3.7. Assume that $\Omega=\left\{z \in \mathbb{C}^{n} ; \rho(z)<0\right\}$ where $\rho \in C^{\infty}(\bar{\Omega})$. For $z_{0} \in \partial \Omega$ suppose that $\left|\nabla \rho\left(z_{0}\right)\right|=1$. Then $\Pi\left(z_{0}\right)$ is the product of the eigenvalues of the form

$$
\sum_{j, k=1}^{n} \frac{\partial^{2} \rho}{\partial z_{j} \partial \bar{z}_{k}}\left(z_{0}\right) \mathrm{d} z_{j} \wedge \mathrm{d} \bar{z}_{k}
$$

restricted to the vector space $\left\{w \in \mathbb{C}^{n} ; \sum_{j=1}^{n} \partial \rho / \partial z_{j}\left(z_{0}\right) w_{j}=0\right\}$. 
Theorem 3.8. Let $\Omega$ be a bounded, strongly pseudoconvex domain in $\mathbb{C}^{n}$ with smooth boundary. Let $f \in C^{\infty}(\bar{\Omega} \times \mathbb{R})$ be a strictly positive function which is increasing in the second variable and satisfies assumptions (A), (B) and (C). For boundary points $z_{0} \in \partial \Omega$ let $\Pi\left(z_{0}\right)$ be defined as in Definition 3.7. Then $u$, any solution to Problem (1), meets the estimate

$$
\lim _{z \rightarrow z_{0}} \frac{\Psi_{n}(u(z))}{d_{\Omega}(z)}=4^{(1 /(n+1))} h\left(z_{0}\right)^{(1 /(n+1))} \Pi\left(z_{0}\right)^{-(1 /(n+1))},
$$

where $z_{0} \in \partial \Omega$.

Proof. After a translation and rotation we can assume that $z_{0}=0$ and that the exterior normal to $\partial \Omega$ at $z_{0}$ is $(1,0, \ldots, 0)$. Doing the same holomorphic coordinate change as in the paragraph after the formulation of Lemma 3.4 we know that there is a $\rho$ so that we can describe $\partial \Omega$ as

$$
\operatorname{Re} z_{1}=\sum_{j, k=1}^{n} \frac{\partial^{2} \rho}{\partial z_{j} \partial \bar{z}_{k}}(0) z_{j} \bar{z}_{k}+o\left(|z|^{2}\right) .
$$

Changing coordinates in the plane $\left\{z \in \mathbb{C}^{n} ; z_{1}=0\right\}$ we can diagonalize the Levi form so that

$$
\frac{\partial^{2} \rho}{\partial z_{j} \partial \bar{z}_{k}}(0)=0
$$

when $j, k \geq 2$ and $j \neq k$. Writing $\rho_{j \bar{k}}=\left(\partial^{2} \rho / \partial z_{j} \partial \bar{z}_{k}\right)(0)$ we make the coordinate change $\zeta_{1}=z_{1}, \zeta_{j}=z_{j}+z_{1}\left(\rho_{1 \bar{j}} / \rho_{j \bar{j}}\right)$ for $j=2, \ldots, n$. In these coordinates $\partial \Omega$ is described as $\operatorname{Re} \zeta_{1}=\widetilde{\rho}_{1 \overline{1}}\left|\zeta_{1}\right|^{2}+\sum_{j=2}^{n} \rho_{j \bar{j}}\left|\zeta_{j}\right|^{2}+o\left(|\zeta|^{2}\right.$ ) where $\widetilde{\rho}_{1 \overline{1}}$ depends on $\rho_{12}, \ldots, \rho_{1 \bar{n}}, \rho_{2 \overline{2}}, \ldots, \rho_{n \bar{n}}$ and it will actually turn out to be irrelevant. Set $\widetilde{\zeta}_{1}=\zeta_{1} \sqrt{\widetilde{\rho}_{1 \overline{1}}}, \widetilde{\zeta}_{2}=\zeta_{2} \sqrt{\rho_{2 \overline{2}}}, \ldots, \widetilde{\zeta}_{n}=\zeta_{n} \sqrt{\rho_{n \bar{n}}}$. In these coordinates the boundary is given by the equation

$$
\frac{1}{\sqrt{\widetilde{\rho}_{1 \overline{1}}}} \operatorname{Re} \widetilde{\zeta}_{1}=\sum_{j=1}^{n}\left|\widetilde{\zeta}_{j}\right|^{2}+o\left(|\widetilde{\zeta}|^{2}\right) .
$$

The equation

$$
\frac{1}{\sqrt{\widetilde{\rho}_{1 \overline{1}}}} \operatorname{Re} \widetilde{\zeta}_{1}=\sum_{j=1}^{n}\left|\widetilde{\zeta}_{j}\right|^{2}
$$

describes a sphere with radius $1 / 2 \sqrt{\widetilde{\rho}_{1 \overline{1}}}$. Given an $\varepsilon>0$ we can find an open neighborhood $U$ of $\widetilde{\zeta}_{0}$ so that the spheres $\left(\sqrt{\widetilde{\rho}_{1 \overline{1}}}-\varepsilon\right)^{-1} \operatorname{Re} \widetilde{\zeta}_{1}=\sum_{j=1}^{n}\left|\widetilde{\zeta}_{j}\right|^{2}$ and $\left(\sqrt{\widetilde{\rho}_{1 \overline{1}}}+\varepsilon\right)^{-1} \operatorname{Re} \widetilde{\zeta}_{1}=\sum_{j=1}^{n}\left|\widetilde{\zeta}_{j}\right|^{2}$ intersect $U \cap \partial \widetilde{\Omega}$ only at $\widetilde{\zeta}_{0}$. Here $\widetilde{\Omega}$ is the image of $\Omega$ under holomorphic coordinate changes above. As in the proof of Proposition 3.6 we can solve a blow-up problem in

$$
\frac{1}{\sqrt{\widetilde{\rho}_{1 \overline{1}}}+\varepsilon} \operatorname{Re} \widetilde{\zeta}_{1}<\sum_{j=1}^{n}\left|\widetilde{\zeta}_{j}\right|^{2}
$$


and

$$
\frac{1}{\sqrt{\widetilde{\rho}_{1 \overline{1}}}-\varepsilon} \operatorname{Re} \widetilde{\zeta}_{1}<\sum_{j=1}^{n}\left|\widetilde{\zeta}_{j}\right|^{2}
$$

with right-hand sides $g(\widetilde{\zeta}) f_{1}(t)$ where $g<h$ and $\tilde{g}(\widetilde{\zeta}) f_{1}(t)$ where $\tilde{g}>h$. Call the solutions $v_{\varepsilon}$ and $w_{\varepsilon}$. Add an affine function to $v_{\varepsilon}$ and subtract such a function from $w_{\varepsilon}$ to get functions $\widetilde{v}_{\varepsilon}$ and $\widetilde{w}_{\varepsilon}$. If we choose the affine functions properly we can use Lemma 1.3 to conclude that $u \leq \widetilde{v}_{\varepsilon}$ in $U \cap\left\{\widetilde{\zeta} \in \mathbb{C}^{n} ;\left(\sqrt{\widetilde{\rho}_{1 \overline{1}}}+\varepsilon\right)^{-1} \operatorname{Re} \widetilde{\zeta}_{1}<\right.$ $\left.\sum_{j=1}^{n}\left|\widetilde{\zeta}_{j}\right|^{2}\right\}$ and $\widetilde{w}_{\varepsilon} \leq u$ in $U \cap \widetilde{\Omega}$. Since adding or subtracting affine functions does not change the blow-up rate we get

$$
\lim _{\widetilde{\zeta} \rightarrow \widetilde{\zeta}_{0}} \frac{\Psi_{n}(u(\widetilde{\zeta}))}{d_{\widetilde{\Omega}}(\widetilde{\zeta})} \leq \lim _{\widetilde{\zeta} \rightarrow \widetilde{\zeta}_{0}} \frac{\Psi_{n}\left(\widetilde{w}_{\varepsilon}(\widetilde{\zeta})\right)}{d_{\widetilde{\Omega}}(\widetilde{\zeta})}=\lim _{\widetilde{\zeta} \rightarrow \widetilde{\zeta}_{0}} \frac{\Psi_{n}\left(w_{\varepsilon}(\widetilde{\zeta})\right)}{d_{\widetilde{\Omega}}(\widetilde{\zeta})}
$$

and

$$
\lim _{\widetilde{\zeta} \rightarrow \widetilde{\zeta}_{0}} \frac{\Psi_{n}(u(\widetilde{\zeta}))}{d_{\widetilde{\Omega}}(\widetilde{\zeta})} \geq \lim _{\widetilde{\zeta} \rightarrow \widetilde{\zeta}_{0}} \frac{\Psi_{n}\left(\widetilde{v}_{\varepsilon}(\widetilde{\zeta})\right)}{d_{\widetilde{\Omega}}(\widetilde{\zeta})}=\lim _{\widetilde{\zeta} \rightarrow \widetilde{\zeta}_{0}} \frac{\Psi_{n}\left(v_{\varepsilon}(\widetilde{\zeta})\right)}{d_{\widetilde{\Omega}}(\widetilde{\zeta})} .
$$

In order to complete the proof we have to analyze in what way the biholomorphisms we have applied changes the right-hand side of our equation and how $d_{\Omega}$ and $d_{\widetilde{\Omega}}$ is related. Since the complex differential of all but the last biholomorphism is the identity at $z_{0}$ we only have to worry about the last transformation. The determinant of the complex differential of the last transformation is

$$
\frac{1}{\sqrt{\widetilde{\rho}_{1 \overline{1}} \prod_{j=2}^{n} \rho_{j \bar{j}}}}=\frac{1}{\sqrt{\widetilde{\rho}_{1 \overline{1}} \Pi\left(z_{0}\right)}}
$$

at $z_{0}$. We have

$$
\lim _{z \rightarrow z_{0}} \frac{\Psi_{n}(u(z))}{d_{\Omega}(z)}=\sqrt{\widetilde{\rho}_{1 \overline{1}}} \lim _{\widetilde{\zeta} \rightarrow \widetilde{\zeta}_{0}} \frac{\Psi_{n}(u(\widetilde{\zeta}))}{d_{\widetilde{\Omega}}(\widetilde{\zeta})} .
$$

This is because close to the boundary point it does not matter whether we measure the distance to the boundary or to the tangent plane. By Proposition 3.6 we get

$$
\begin{aligned}
& 2\left(\frac{1}{\widetilde{\rho}_{1 \overline{1}} \Pi\left(z_{0}\right)}\right)^{(1 /(n+1))} h\left(\widetilde{\zeta}_{0}\right)^{(1 /(n+1))}\left(\frac{1}{2\left(\sqrt{\widetilde{\rho}_{1 \overline{1}}}+\varepsilon\right)}\right)^{(n-1 /(n+1))} \leq \lim _{\widetilde{\zeta} \rightarrow \widetilde{\zeta}_{0}} \frac{\Psi_{n}(u(\widetilde{\zeta}))}{d_{\widetilde{\Omega}}(\widetilde{\zeta})} \\
& \leq 2\left(\frac{1}{\widetilde{\rho}_{1 \overline{1}} \Pi\left(z_{0}\right)}\right)^{(1 /(n+1))} h\left(\widetilde{\zeta}_{0}\right)^{(1 /(n+1))}\left(\frac{1}{2\left(\sqrt{\tilde{\rho}_{1 \overline{1}}}-\varepsilon\right)}\right)^{(n-1 /(n+1))} .
\end{aligned}
$$

Let $\varepsilon$ tend to zero and get

$$
\lim _{\widetilde{\zeta} \rightarrow \widetilde{\zeta}_{0}} \frac{\Psi_{n}(u(\widetilde{\zeta}))}{d_{\widetilde{\Omega}}(\widetilde{\zeta})}=2\left(\frac{1}{\widetilde{\rho}_{1 \overline{1}} \Pi\left(z_{0}\right)}\right)^{(1 /(n+1))} h\left(\widetilde{\zeta}_{0}\right)^{(1 /(n+1))}\left(\frac{1}{2 \sqrt{\widetilde{\rho}_{1 \overline{1}}}}\right)^{(n-1 /(n+1))} .
$$


Hence

$$
\begin{aligned}
\lim _{z \rightarrow z_{0}} \frac{\Psi_{n}(u(z))}{d_{\Omega}(z)} & =\left(\frac{1}{\widetilde{\rho}_{1 \overline{1}} \Pi\left(z_{0}\right)}\right)^{(1 /(n+1))} h\left(\widetilde{\zeta}_{0}\right)^{\frac{1}{n+1}}\left(2 \sqrt{\widetilde{\rho}_{1 \overline{1}}}\right)^{1-(1 /(n+1))} \\
& =\left(\frac{4}{\Pi\left(z_{0}\right)}\right)^{(1 /(n+1))} h\left(\widetilde{\zeta}_{0}\right)^{(1 /(n+1))} \\
& =\left(\frac{4}{\Pi\left(z_{0}\right)}\right)^{(1 /(n+1))} h\left(z_{0}\right)^{(1 /(n+1))}
\end{aligned}
$$

\section{Boundary behavior of the Bergman kernel}

In this section we shall apply our results on the blow-up rate of solutions to MongeAmpère equations to describe the asymptotic behavior of Bergman kernel. These results are known and in [5] Hörmander obtained a more general result which also holds for weighted Bergman kernels. We first recall the definition of the Bergman kernel and some basic results. A reference for this is [9].

Let $\Omega$ be a domain in $\mathbb{C}^{n}$. We call $\mathcal{O} L^{2}(\Omega)=\mathcal{O}(\Omega) \cap L^{2}(\Omega)$ the Bergman space. Given a compact subset $K$ of $\Omega$ one can show that there is a constant $C_{K}$ such that

$$
\sup (|f(z)| ; z \in K) \leq C_{K}\|f\|_{L^{2}(\Omega)}
$$

for all $f \in \mathcal{O} L^{2}(\Omega)$. This inequality yields that $\mathcal{O} L^{2}(\Omega)$ equipped with the inner product $\langle f, g\rangle=\int_{\Omega} f(z) \overline{g(z)} \mathrm{d} \lambda(z)$ is complete and hence a Hilbert space. The inequality also gives that the functionals, one for each $z \in \Omega, \Phi_{z}(f)=f(z)$ are bounded linear functionals. The Riesz Representation Theorem guarantees that there is $k_{z} \in \mathcal{O} L^{2}(\Omega)$ such that

$$
\Phi_{z}(f)=f(z)=\left\langle f, k_{z}\right\rangle .
$$

The Bergman kernel is the function $K(z, \zeta)=\overline{k_{z}(\zeta)}$. It can be shown that, for a domain $\Omega \Subset \mathbb{C}^{n}$, we have

$$
K(z, z)=\sup \left(|f(z)|^{2} ; \quad f \in \mathcal{O} L^{2}(\Omega),\|f\|_{L^{2}(\Omega)}=1\right) .
$$

In [4] Fefferman showed that the asymptotic behavior of $K(z, z)$ as $z \rightarrow z_{0}$ for $z_{0} \in \partial \Omega$ is the same as the boundary behavior of $\left(n ! / \pi^{n}\right) e^{(n+1) u(z)}$ where $u$ is the solution to

$$
\left\{\begin{array}{l}
\operatorname{det}\left(\frac{\partial^{2} u}{\partial z_{j} \partial \bar{z}_{k}}\right)=\mathrm{e}^{(n+1) u(z)} \text { in } \Omega \\
\lim _{z \rightarrow z_{0}} u(z)=\infty \text { for all } z_{0} \in \partial \Omega .
\end{array}\right.
$$

With the same notation as in Theorem 3.8 we see that

$$
\lim _{z \rightarrow z_{0}} \frac{\Psi_{n}(u(z))}{d_{\Omega}(z)}=\left(\frac{4}{\Pi\left(z_{0}\right)}\right)^{(1 /(n+1))}
$$


for $z_{0} \in \partial \Omega$. We have to calculate, or at least estimate,

$$
\Psi_{n}(u)=\int_{u}^{\infty}\left(\mathrm{e}^{(n+1) t}-1\right)^{-(1 /(n+1))} \mathrm{d} t .
$$

Since

$$
\lim _{t \rightarrow \infty} \frac{\mathrm{e}^{(n+1) t}}{\left(\mathrm{e}^{(n+1) t}-1\right)^{(1 /(n+1))}}=1
$$

we get, for arbitrary fixed $\varepsilon>0$ and $u$ is large enough,

$$
\mathrm{e}^{-u} \leq \Psi_{n}(u) \leq(1+\varepsilon) \mathrm{e}^{-u} .
$$

This yields

$$
\mathrm{e}^{-u(z)} \leq d_{\Omega}(z)\left(\left(\frac{4}{\Pi\left(z_{0}\right)}\right)^{(1 /(n+1))}+\varepsilon\right)
$$

and

$$
d_{\Omega}(z)\left(\left(\frac{4}{\Pi\left(z_{0}\right)}\right)^{(1 /(n+1))}-\varepsilon\right) \leq(1+\varepsilon) \mathrm{e}^{-u(z)}
$$

when $u(z)$ is large enough. Thus for $z$ close enough to $z_{0}$ we get

$$
\begin{aligned}
\left(\left(\frac{4}{\Pi\left(z_{0}\right)}\right)^{\frac{1}{n+1}}+\varepsilon\right)^{-(n+1)} & \leq d_{\Omega}(z)^{n+1} \mathrm{e}^{(n+1) u(z)} \\
& \leq(1+\varepsilon)^{n+1}\left(\left(\frac{4}{\Pi\left(z_{0}\right)}\right)^{(1 /(n+1))}-\varepsilon\right)^{-(n+1)}
\end{aligned}
$$

which yields

$$
\lim _{z \rightarrow z_{0}} d_{\Omega}(z)^{n+1} \mathrm{e}^{(n+1) u(z)}=\frac{\Pi\left(z_{0}\right)}{4} .
$$

We have proven the following result.

Theorem 4.1. Assume that $\Omega$ is a bounded strongly pseudoconvex domain with smooth boundary. Let $K_{\Omega}(z, w)$ be the Bergman kernel of $\Omega$. For boundary points $z_{0}$ let $\Pi\left(z_{0}\right)$ be as in Definition 3.7. Then

$$
\lim _{z \rightarrow z_{0}} d_{\Omega}(z)^{n+1} K_{\Omega}(z, z)=\frac{n !}{4 \pi^{n}} \Pi\left(z_{0}\right)
$$

for all $z_{0} \in \partial \Omega$.

Acknowledgements. We would like to thank Vilhelm Adolfsson and Bo Berndtsson who told the second author about the connection between boundary blow-up problems for the complex Monge-Ampère operator and the Bergman kernel. 


\section{References}

[1] Błocki, Z.: Interior regularity of the complex Monge-Ampère equation in convex domains. Duke Math. J. 105, 167-181 (2000)

[2] Caffarelli, L., Kohn, J. J., Nirenberg, L., Spruck, J.: The Dirichlet problem for nonlinear second order elliptic equations, II. Complex Monge-Ampère, and uniformly elliptic, equations. Commun. Pure Appl. Math. 38, 209-252 (1985)

[3] Cheng, S. Y., Yau, S. T.: On the existence of a complete Kähler metric on non-compact complex manifolds and the regularity of Fefferman's equation. Commun. Pure Appl. Math. 33, 507-544 (1980)

[4] Fefferman, C.: Monge-Ampère equations, the Bergman kernel, and geometry of pseudoconvex domains. Ann. Math. 103, 395-416 (1976)

[5] Hörmander, L.: $L^{2}$ estimates and existence theorems for the $\bar{\partial}$ operator. Acta Math. 113, 89-152 (1965)

[6] Ivarsson, B.: Interior regularity of solutions to a complex Monge-Ampère equation. Ark. Mat. 40, 275-300 (2002)

[7] Ivarsson, B.: Regularity and uniqueness of solutions to boundary blow-up problems for the complex Monge-Ampère operator (submitted for publication, 2005) $14 \mathrm{pp}$

[8] Kobayashi, S.: Differential Geometry of Complex Vector Bundles. Princeton University Press (1987)

[9] Krantz, S. G.: Function Theory of Several Complex Variables, 2nd edn. Princeton, Wadsworth \& Brooks (1992)

[10] Matero, J.: The Bieberbach-Rademacher problem for the Monge-Ampère operator. Manuscripta Math. 91, 379-391 (1996) 